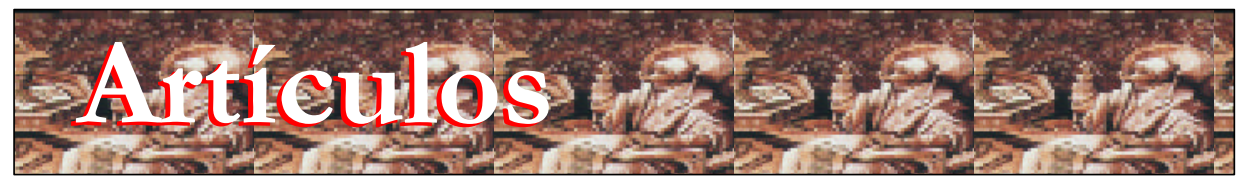

\title{
Marco curricular de los estudios cuantitativos de la información en biblioteconomía y documentación en España
}

\author{
MARÍA J. REYES-BARRAGÁN \\ Facul taddeBiblio teconomíayDocumen ta cióndelaUniversidad \\ deExtremadura C/ Alcazabas/n06011Badajoz \\ E-mail:mjreyes@alcazaba.unex.es \\ CRISTINA LÓPEZ-PUjALTE \\ Facultad de Biblioteconomía y Documentaciónde la Universidad \\ de ExtremaduraC/Alcazabas/n06011Badajoz \\ E-mail:clopez@alcazaba.unex.es

\section{VICENTE GUERRERO BOTE} \\ Facul taddeBiblio teconomíayDocumentacióndelaUniversidad \\ deExtremadura C/ Alcazabas/n06011Badajoz \\ E-mail: vicente@alcazaba.unex.es
}

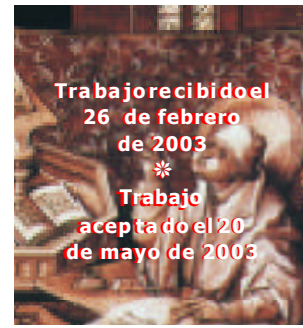

\section{RESUMEN}

Partiendodeldesarrollodelos es tudios debiblioteconomíaydocumentación (ByD) en Es paña, ana li za mos la pre sen cia de la biblio me tría y de las ma te rias decortecuantitativo enlos planes dees tudiodeByDvigen tes actualmenteen España.

Para ello nos he mos cen tra do en la di plo ma tu ra en $\mathrm{ByD}$, li cen cia tu ra en do cur mentaciónydocto rado, yseleccionadolas asigna tu ras rela ciona das con as pectos cuantitativos a la vez que hemos estudiado la categorización de éstas, y mos tramos elbalance final delproce so derenovación delos planes de es tudioy delas ma te rias rela cio na das con la cuan ti fica ción delos proce sos in for ma cio nales y el posicionamiento de las distintas escuelas y facultades en España.

Palabras Clave: Biblioteconomía; Documentación;España; Planes de estudio; Bibliometría; Materiascuantitativas 


\author{
CURRICULAR FRAMEWORK FOR QUANTITATIVE STUDIES OF INFORMATION \\ IN LIBRARY SCIENCE AND DOCUMENTATION IN SPAIN \\ MARÍA J. REYES-BARRAGÁN \\ CRISTINA LóPEZ-PUjALTE \\ VICENTE GUERRERO-BOTE \\ FacultaddeBiblio te conomíayDocumen tación delaUniversidad \\ deEx tremadu ra C/Alcazabas/n06011 Badajoz \\ E-mail:vicente@alcazaba.unex.es
}

\begin{abstract}
We analyze the presence of bibliometrics and other quantitative subjects in the L\&IS curricula presently in force in Spain. Our focus was on L\&IS diploma course, the information science degree and the $\mathrm{PhD}$ courses. We selected the categorization of subjects related with quantitative aspects and show the final renewal process of the curricula and the subjects related with the quantification processes of information and the positioning of the different schools and faculties of Spain.

Key Words: Library Science; Information Science; Spain; Study Plans; Bibliometrics; QuantitativeSubjects.
\end{abstract}

\title{
INTRODUCCIÓN
}

$\mathbf{L}^{2}$ aaplicación demé to dos matemáticosyestadísticossehavenidohaciendodesde finales del S. XIX en diversos campos del conocimiento como la economía, la sociología, la biología, la psicología, etcétera.

Esteproceso dematematizacióntambién sehaproducidoenlacienciadela docut men ta ción y en aquellas dis ciplinas que hanido ge ne ran do una se rie de es pecialida desmétricasdentrode disciplinasquedesarrollanun marcoteóricopropio.

Los principios teóricos y metodológicos de los estudios métricos de la informaciónsonabordadosensuestudioporGorbea Portal(1994).

Elpresen te trabajo aco me te los es tu dios métricos delain forma ción en el marco curriculardelabibliotecono míayla documentación(ByD)españoles,ydeterminasu pre sencia y el peso que és tos tie nen den tro de los planes dees tu diodelas res pec tivas facultadesyescuelasdeByD.

Sin em bargo, com pren der el de sa rrollo eim plan ta ción dela biblio me tría y delos es tudios cuan titativos nos lle vaa reflexionarcómo sedesarrolla ronla formaciónde bibliotecarios ydocumentalis tas yel contex to curricularenEs paña. Tambiénse analizan los pla nes de es tu dio en $\mathrm{By}$, ubi can do en ellos la biblio me tría y otras asig na tur ras de cortecuantitativo que presentan distin toscalificativos debidoala au tonomía universita ria, pues se tra ta de ma te rias de re cien teim plan ta ción. Se es tu dia la di plomatura, lalicencia turayeldoctora doapartirdelos planesdees tu diosac tualmente vigentesenEspaña. 
Creemosquela promocióndees tudioscuan titativos debeseruna prácticacomún enla enseñanzadeByDyentrebibliotecariosydocumentalis tas, porqeenospermitende tectar ten dencias enlos flu jos dein forma ción, tan toenla ge ne ra ción de conocimien to científico como actividad integrada en la gestión de unidades de información, como en el mejora mientodesueficacia,calidadylasatisfaccióndelasdemandassociales.

\section{CONTEXTO CURRICULAR EN ESPAÑA}

Antes de seralberga da porlaUniver sidadla forma ción de profe sio nales bibliotecarios-documentalistasenEspaña, secaracterizóporserautodidacta.

Unhechoatenerencuenta enla formación debiblio tecarios-documentalis tas fueel granvolumen de do cu men ta ción que pasóa ma nos delEs ta do me dian telas políticas desamortizadorasdeMendizabalyMadozdurantelaprimeramitaddelsiglo XIX. Esto hizo necesaria la formación de personal capacitado para conservar y custodiar estos ma te ria les y, con tal mo tivo, se pro mulgó una Real Or den el 7 de oc tu bre de 1856 que creóla Es cuelaSuperiordeDiplomática, cuyoobje tivo principal se ríala forma ción de dichopersonal.La en señanza seim partiría en tres cursos yla titula ción ob teni dacapacitaba paraingre sarenelcuerpo Faculta tivo de Archive rosy Bibliote ca rios, cuyacreaciónsellevóacabocasicontemporáneamentealaEscuela(1858).

Como se in tu ye de esta primera fun da ción, la en se ñan za ofi cial de es tu dios debiblio te conomíanace delane cesidad de aco me terta reas propias delas ac tivida des del mundo de las bibliotecas: control, inventario, conservación y, en menor medida, puestaadisposicióndelma terialdocumentalybibliográficodelquesedisponía.

En1900desa pare celaEs cuelayla en señanza seráasumida con res tric ciones drás ticas porla Fa cul tad de Filoso fíayLetras dela UniversidadCen traldeMadrid, yaque en el plan de es tu dios de di cha Fa cul tad sólo exis tía una asig na tu ra lla ma da bi bliología y más tarde biblio gra fía. Tras diversosava ta resinclu soestaasigna tu radesapareció de los pla nes de es tu dio y du ran te un buen perio do de tiem pola univer si dad es pa ñola no aten dió, ni siquie ra mo des ta men te, nada que tu vie ra rela ción con el mun do delosarchivosylasbibliotecas.

En 1956laUniversidad Complutenseiniciala docencia dela asigna tu ra Bibliogra fíahispánicayMetodologíadelainvestigación.

Fue raya delám bi to delauniversidad se es ta blecie ron hace mu chos años va ria dos cursosparala formacióntécnicadearchiverosybibliotecarios:

* Eldenominadocursopara la Forma ción Téc nica de Archive ros Biblio teca rios y Ar queó lo gos, fue crea do en Ma drid en 1952 y dio lu gar a la Es cue la de Documentalistas, lacualdependióorgánicamenteprimerodelMinisteriode Educación y Ciencia, y posteriormente del Ministerio de Cultura. En 1980 cambió de nombre para denominarse Centro de Estudios Bibliográficos y Documentarios (CEBYD) con carácter de Centro de Investigación. 
Escola de Biblioteca ries na cida para aten derlas ne cesida des delas Bibliotecas Populares de Cataluña. Este centro permaneció en vigor desde su creación en 1915 has ta que se con vir tió en 1981 en la es cue la universi ta ria "Jor di Rubió i Balaguer" de Biblioteconomía y Documentación adscrita a la Universidad de Barcelona.

También se crea la Escuela de Bibliotecarios de la Universidad de Navarra en 1967.Lamentablemente sólo tuvouna déca da defuncionamien to; suses tudios sees tructuraronentrescursos.

Paralelamente diversas facultades han incluido asignaturas tales como la paleogra fía, la biblio te conomía, la biblio lo gía, etcéte ra, en tre las de geo gra fía ehis to ria de diver sas univer si da des. Perola de más re cien te crea ción es la de Cien cias dela in for mación, que prácticamenteestáin cluidaen to doslos planesdees tudiodelas diversas universidades.

Delovis to has ta ahora pue de de du cirse quelos es tu dios de ByD hantenidounca rácteranexoala en señanzauniversi ta ria yque éstalos ha he cho suyos sólo parcialo, a veces, intermitentemente.Sinembargolauniversidadespañolahareaccionadotarde ylen ta men te pero con una res pues taglo bal ein ten saaloquela so cie dadle de man da ba vivamen te: for marpro fe sio nales con cate go ría universita ria para el mun dodela documenta ción. Estares pues ta se plas may con cre ta en la crea ción delas ac tua les di plomaturas en biblioteconomía y documentación, y la licenciatura en documentación hace poco más de dos déca das, fe cha tar día sise com pa ra con elám bi to an glo sa jóndondelasescuelasllevanmásdeunsiglodehaberseestablecido.

ElRealDe cre to 3.104/78 de 1 de di ciem bre,BOE(Bole tínOficial delEstado) del 9deenerode1979, crealoses tudios universita rios debiblioteconomíaydocumenta ción, permi tien do quelas dis tin tas universi da des pu dieran elaborar sus planes deestu dioy solicitarlacrea ción de cen tros. Nacen asílas prime ras escuelasuniversitarias, ladeBarcelona (RubióiBa la guer) ylade Granada (1982) a las que paula tinamentese iránincorporando, unavezcumplidoslos preceptivos trámi tes de aprobación depla nes, las de Sa la man ca (1987), Murcia (1988), Za rago za (1989), León(1990), CarlosIII y Complutense (1990), San Pablo (CEU) (1995), Extremadura (1994), La Coruña y Valencia(1996).

LaaprobacióndelaLey deReformaUniversita ria(II/1983)suponeunimportantecambioenlasestructurasacadémicasyproducelaadecuaciónde diversosaspectos deltrabajouniversitarioalasnuevasnormativas.

La reforma de los planes de estudio de la diplomatura la podemos encontrar en (Delgado López-Cozar, 1992a). Y la situación de la documentación en España ha sido expuesta por Aba dal en su te sis doc to ral tan to des de el pun to de vis ta de la forma ción como dela pro fe sión (Aba dal,1994). Laim plan ta ción del se gun do ci clo universitariosepuedeencontraren(LópezYepes,1995).

Engenerallain troducción deloses tudios cuan tita tivos enlosplanes deestudiode las es cuelas y fa cul ta des de ByD ha sido un proce so paula tino y to da vía se en cuen tra enfasedeimplantación. 
Sin em bargola preocupa ción, tan to a nivel na cio nal como in terna cio nal,porlos fun da men tos cien tí fi cos de la ges tión de la in for ma ción vino apa re ja da de una profunda revolución tecnológica que su pu so en Es pa ñala su pe ra ción del per fil hu ma nista del bibliotecario-conservador de documentos, y lo sustituyó por la del documentalista-suministrador de información a quien se le pretendía imponer un perfil queleproporcionaralosconocimientosteóricosquesubyaceneneldesarrollodesus tareas. Untécnicoca pazdeloca lizary pro ce sar in for ma ción. Sinembargo, en Es pa ñaelperfilhuma nistaten deráa prevale cercuandoaparecenlasprimerasescuelasd e biblioteconomía.

Dehe choenlos prime ros pla nes de es tu dios las asignaturas de cor te cuan tita tivo no estaban presentes; lastecnologías delain formacióncomenzabanadesarrollarse al igual que la cien cia dela in for ma ción, o la de la do cu men ta ción, y és tas eran con cebidasmásquecomounmétodocientíficocomounconjuntodetécnicas.

A principios de los años 90, con las escuelas en plenoprocesodeconsolidación, los profesionalesde las bibliotecas re cha zaban la utilidad deloses tu dios universitarios es pecializados de $\mathrm{ByD}$, aun que re co no cían el ciclo de postgra do (Apa ricio Fernández,1990).

En biblio te cono míay docu men tación hanocurrido dos he chosquehanin fluido en ellas de formatrans ce dental. Es tos son: elde sa rrollodelas nuevas tec no logías yla concepción delain forma ción comorecursoinago table, fle xibley moldeableque sur ponelautilización detécnicasy cono cimien tos dis tin tosalos utiliza dos tra dicionalmenteparalagestióndeestosrecursos(Sanz,2001).

En cuanto a la formación de los profesionales de la información, Félix de Moya analizalaformación de es tos fu tu ros profe sionalesyseñalaloscambiosqueéstosd ebenexperimentar(Moya,1996):

* Será un usuario experto de la tecnología de la información, ya que su labor profesional estará ligada al manejo de los recursos tecnológicos, por lo que difícilmente podrá prescindir de ellos para realizar cualquier tarea.

* Su actividad fundamental estará relacionada con su papel de intermediario. Es de cir, suan tigua función de re cep tory con serva dor de do cu men tos, mur chas ve ces como elúltimoesla bóndelacadena documen tal, se trans forma rá en la de un intermediario que desempeña su actividad aproximando información y usuarios.

* En re la ción con lo an te rior, la aten ción y la for ma ción de los usua rios se rán actividades a las que dedicará la mayor parte de su tiempo y trabajo.

- Seráasi mis mo uncuan tifica dor de cos tos y ren dimientosy, en de finitiva, un evaluador.

* Será un profesional más abierto al mercado que al producto y estará mejor informado de aquello que el mercado ofrece para ponerlo a disposición de los usuarios. 
El trabajo rea li za do por Camps y Cres pán(1997) efec túa un es tu dio de merca do con el fin de co no cer la opi nión de los di rec tivos de las em pre sas en tor no a las cuestio nes siguien tes: ellu garque ocu pa la in for ma ción en la es tra te giage ne ral dela empresa;lassolucionesadoptadaspara re solverlosproble masplanteadosporlagestión de la información, y el perfil del profesional que, en su opinión, puede resolver los problemas reales. Elanálisis delos da tos demos trólaimportan ciaquelasempresasle daban alain for ma ción y la ne ce sidad de ges tio narla co rrec ta mente. Sin em bargo en numerosasocasioneslas solucionesadoptadaserandecaráctertecnológico.

En la mis ma línea (John son, 1998) in cide en los cam bios pro du ci dos por la so ciedad dela in for ma cióny su in fluen ciaen el papelque de ben de sem pe ñarlos pro fe sionales, ein sis teen quelas es cuelas de biblio te co nomíaydocu men ta ción de ben seguir uncaminoparaleloaldelmercadolaboral.

Gorman(1999)señalaqueanivelprofe sional hayaspectosquenocambian, mientrasqueotros requie ren mayorniveldeespecialización. Opinaquelasmejoresescuelassonaquellasquesehandecididoporuncurrículummásamplio.

Has ta hace poco tiem polas ad mi nis tra cio nes pú bli cas eran las que ofre cían más pues tos de trabajo, si tua ción corro boradaenel estu diorealizadopor Mo reiro, Mos co so y Or tiz (1995), cu yos da tos re ve lan que el sec tor pú bli co es el que más em pleo genera, con ci fras que os cila ban en tre el $90 \%$ y el $79 \%$, fren te al $16 \%$ del sec tor privado.

Sinembargolainterdisciplinariedaddelosactualesplanesdees tudioespañolesh a repercutidoen las salidas profesionales de los graduadosque encuen tran trabajo en ámbitosdis tin tosaldelabiblio teconomíayla documenta ción. Así,pare cequelatendenciahacialas salidaslaborales estácambiando; enestalínea sepronuncia el estudio realizadoporMoreiroyCaridad(1998).

LópezYepes (1996) señalaquela figuradel profe sionaldelain formacióndeberedefinirsepermanentementeapartirdelossiguientesfactores:

* El cambio social.

* Las tendencias formativas existentes en sociedades más avanzadas que la nuestra.

* El análisis comparativo de los planes de estudio vigentes.

* La con figura ción del papel so cialdel pro fe sionalenEs paña,en rela cióncon el mercado de trabajo.

- El mercado de trabajo y las propuestas de nuevas necesidades.

Si la institucionalizaciónde la disciplina en España fue tardía, la investigación se encuentraenestadoembrionario, seincorporaalaactividadinvestigadoraenladécada de los 80 , y to da vía ha brá que es pe rar has ta la dé ca da de los 90 para que se pro duzcan los primeros trabajos empíricos sobre la investigación (Roman \& Sorli, 1994; Abadal,1994;LópezLó pez,1996; JiménezContreras, 1997; Pérez Álvarez-Oso rio, 1997; Moya Anegon et al., 1998; Frías, 1998; Del ga do Ló pez Co zar, 2000, 2002; LópezYepes,2001yZapicoAlonso,2002). 


\section{JUSTIFICACIÓN DE LAS MATERIASCUANTITATIVAS EN LOS PLANES DE ESTUDIODE BYD EN ESPAÑA}

Elenormede sarrolloquela cien cia ha te nidodes de me dia dos delsiglopa sa dovieneajus tificarquelaactividadbiblio tecariaycientífico-informativahayaido configurando, durante toda su trayectoria de surgimien toydesarrollo, unsis tema de conocimiento integrado por materias que con un marco teórico propio abarcan a su vez mé to dos y téc nicas quele dan res pues ta a su fun ción y a su ob je to. Esto pro du ce, necesariamente, un doble efecto de vertebraciónyparcelación deconocimien tos que has ta estemomento se hallaban dis persos y delos cuales formaunaim por tan te parte lo que podríamos llamar "metrías" (Biblio-Ciencio-Infor-metría), y que nos harán hablar,portan to, del na cimien to de jóve nes dis ci pli nas que ha cen suyos los es tu dios mé tricos delain forma ción, y sobrelas que ha bre mos de tener encuen ta que abor dan ma te rias cu yas es truc tu ras pro pias es tán en proce so de for ma liza ción o cuan do menosde afian za mien to, y por tan to to da vía es di fícilaco tarlas y, más di fícilaún, de finir perfectamen tesus fron teras con cien cias afines conlas que com parten me to dologías semejantes.

Enelcasodelabiblio metría elcaminore corrido ha sidoes pecialmentesignificativo, ya quelos prime ros tra ba jos so bre el tema son realiza dos con téc nicas ma te má ticas muy básicas para posteriormente caer en una excesiva matematización que en ocasiones hacon fun didoelfinconelmedio, oconvertido, tambiénequivoca damente, elmedioenun fin ensímis mo.

Sin embargo, es hora ya de que el abordaje de la bibliometría, como un método científicodelaCienciadelaInformación, empieceallevarseacaboporprofesionales bibliotecarios que hayan recibido una for ma ción cuan do me nos bá sica so bre el conocimien to de su mar co teó ri co y delas múl tiples apli ca ciones prác ticas que su de sa rrollo puede proporcionar, así como de la metodología y herramientas necesarias parallevarlas acabo.

Es necesa rio, porotraparte, lle garaun pun to de en cuen troco mún en la dis tan cia que tradicionalmente ha separado los estudios universitarios denominados "ciencias", seanéstas exactas o experimentales, y"letras", con side ra das como humanida des que pro du cen como con se cuen cia inelu dible, ti tu la dos que huyen de la cuan tiffcación de los fenómenos, y otros que, en estudios de evaluación matemática o estadística,ignoranabsolutamentelosmáselementalesprincipiosconceptuales.

Sehace en ton ces in dis pensable que te rrito rios in terme dios en trelas cien cias ylas le tras sean cultiva dos porprofesionales que reú nan ambas forma ciones para nocaer en graves erro res en cuan to a fi na li dad o meto do lo gía. Porotra par te para el biblioteca rio o documen talis ta se ha cen cada vez más ne ce sa rios co no ci mien tos so brepará me tros y variables rela cio na dos conla pro ducción cien tífica, su circula ción y suconsumo, independientemente de los soportes en que ésta pueda recogerse (Reyes Barragán,1997). 
La re forma delos pla nes dees tu dio a la que alu dia mos an te rior men te sig ni ficóla opor tu nidad de com ple tar los tres ciclos universita rios con la pues ta en mar cha de la licencia tura, aunqueeltercercicloseimpartíaenalgunasuniversida desyexis tía la posibilidadderenovarconteni dos. Autores comoJiménez Contre ras (1990,1998);Ferreiro Aláez, (1990); Amat (1991); Delgado López-Cozar (1992b); Sebastiá Salat (1992)Currás (1993);LópezLó pez(1995), señala banlanecesidad dein corporarma teriasrelaciona dasconlos fundamentoscien tíficos dela dis ciplina.Las nuevas materiasin corpo ra das tenían que verconlas tecnologías delain forma ciónyla es ta dís tica, aun que ésta sólo en el ci clo de li cen cia tu ra, pero no con tem pló a la bi blio me tría por considerar que los conceptos que manejaba presentaban dificultades para los estudian tes y que ésta se guía sien do una des co no cida. Tam po co la cien cia de la in formación tuvoacogidaenlosnuevosplanes.

Encierta forma, ladi fu sión dela biblio me tría es ta ba di ficul ta da porqueno se dispo níadeunasis te ma tización de ca rác terdo cente, nidelos conocimientos, niexis tía una terminología aceptada, ni tampoco se había estructurado una metodología biblio mé trica de base cien tífica, ni mu cho menos una di dác tica acorde con todo ello. A esto ha bía que aña dircómo eran abor da dos los te mas biblio mé tri cos en la li te ra tu ra especializada,pueslasmás delasvecesés tos resul tabaninacce sibles parabibliotecariosy documentalis tas conuna formación prácticamen tehumanís ticapeseaserprecisamen teellos quienesen buena lógica de be rían sersus des tina tariosnaturales(FerreiroAláez,1987).

Eldesarrollodelabibliometría, in forme tríayotrasmate rias decortecuan titativo enlos países an glo sajonesy eu ro peos, se abor dan enlos es tu dios realizados porCronin (1992); Prevot (1990); Meyriat (1993); Ingwersen (1994) y Jiménez Contreras (1998).

Sin em bargo, el ca mino delas nue vas tec no logías ha sido se gui do por nu me ro sas escuelasdebiblioteconomíaydocumentaciónnorteamericanas (California,Los Angeles, UCLA; Ber ke ley o In dia na), y otras se han orien ta do más ha cia la ges tión de recursos informativos (Siracusa, Maryland), pero am bos ca sos han su pues tola in corporación de conocimientos procedentes de otras disciplinas y una adaptación a las nuevasnecesidades sociales. Algo si mi larocu rrió en el Rei no Uni do, don de se produ jo una adap ta ción de su currícu lay se in cor po ra ron en sus progra mas tec no logías de la in for ma ción, ges tión y el tra ta mien to cuan ti ta ti vo de da tos, a la vez que sur gió unaorien tacióngeneral hacia elmundodelosnegocios, laeconomíaylacontabilidad (Sanz, 2001).

Encuanto alainves tiga ción basada en métodoscuan titativos,ésta seceñíacasiexclusivamente al CSIC(Con sejo Su perior deInves tiga cio nes Científicas) yera recha zada por los profesores delas escuelas porsuaspec to excesivamen tenu mérico.De he chocuan do seini cióla re forma, labiblio me tría y otras ma te rias cuan ti ta tivas eran prácticamente des conocidas yen los planes dees tu dioque da ron re ducidas alaes tadísticayatécnicasdocumentalesaplicadasalainvestigación. 


\section{LAS MATERIASCUANTITATIVAS EN LOS CURRÍCULADE BYD}

La Reforma de las Enseñanzas Universitarias (LRU/1983) obligó a modificar la es truc tu ra de to dos los pla nes de es tu dio. Tras el Real De cre to 1497/1987 del 27 de noviembre semarcanlas direc trices gene ra les delospla nes de es tu dios ydelos títulos universitarios de carácteroficial, locualin fluyeen to das lascarreras universita rias españolasylasobligaareformarcadaunadelastitulaciones.

Lareformaes truc turalas materias en créditos, una nuevaunidad demedidaquese traduce en horas. Los planes, ya reformados, incluyen los conceptos de materias troncales (deobliga do cum plimien to para to daslas universida des); obliga to rias (es tablecidas por cada univer si dad a par tir de las tron cales), y op ta tivas y de li bre elección. Por tanto, la reforma establece que los planes de estudios deben ser cíclicos, modularesyflexibles.

Cíclicos, dado que, se gún el ar tículo 30 de laLRU,loses tu diosuniversita rios se estructurarán en un máximo de tres ciclos.

* Modulares. Cada asignatura tendrá una carga lectiva en créditos. Para obtener un título el alumno deberá haber cursado y superado una determinada cantidad de créditos.

* Flexibles. El alumno ten drá de re cho a con figu rar su pro pio per fil aca démico, con el menor número posible de limitaciones; de este modo cada estudiante podrá elaborar su propio currículum, en función de las asignaturas optativas y de libre elección que seleccione.

Lasnue vas direc trices es ta ble cen las siguien tes ma te rias (ta bla 1), como componentes básicos del título de diplomado en biblioteconomía y documentación (Real Decreto1422/1991):

Tabla1.Materiasbásicasdeldiplomadoenbiblioteconomíaydocumentación

\begin{tabular}{|l|l|}
\hline \multicolumn{1}{|c|}{ MATERIAS TRONCALES } & \multicolumn{1}{c|}{ DESCRIPCIÓN } \\
\hline $\begin{array}{l}\text { Análisis y lenguajes documentales } \\
\text { (20 créditos) }\end{array}$ & $\begin{array}{l}\text { Introducción a la catalogacióny clasificación. Tratamiento y } \\
\text { recuperación de la información. Descripción bibliográfica de } \\
\text { documentos en distintos soportes, acceso a la descripción y } \\
\text { confección de catálogos. Teo ría y es truc tu ra delas cla si fi ca } \\
\text { ciones y sistemasdeindización. Análisis decontenido }\end{array}$ \\
\hline $\begin{array}{l}\text { Archivística } \\
\text { (10 créditos) }\end{array}$ & $\begin{array}{l}\text { Introducción al estudio y organización de los archi vos. Nor } \\
\text { mas para la conservación,organización y descripción de los } \\
\text { fondos documentales. Funciones y servicios dearchiveros }\end{array}$ \\
\hline $\begin{array}{l}\text { Bibliogra fía y fuen tes dein forma } \\
\text { ción (10 créditos) }\end{array}$ & $\begin{array}{l}\text { Naturaleza,función y tipología delas fuen tes documen tales, } \\
\text { tanto generales como especializadas, estudio histórico y evo- } \\
\text { lutivo de la bibliografia. Historia del libro impreso. Reperto- } \\
\text { rios bibliográficos y metodología de su elaboración. }\end{array}$ \\
\hline
\end{tabular}


Tabla1.Materiasbásicasdeldiplomadoenbiblioteconomíaydocumentación

(Cont.)

\begin{tabular}{|c|c|}
\hline MATERIAS TRONCALES & DESCRIPCIÓN \\
\hline $\begin{array}{l}\text { Biblioteconomía } \\
\text { (10créditos) }\end{array}$ & $\begin{array}{l}\text { Organizacióny administración de bibliotecas yhemerotecas. } \\
\text { Edificios e instalaciones. Conservación y res tau ración. Servi } \\
\text { cios de ex ten sión al usuario. Sis te mas na cio nales ein ter na cio- } \\
\text { nales de bibliotecas }\end{array}$ \\
\hline $\begin{array}{l}\text { Documentacióngeneral } \\
\text { (10créditos) }\end{array}$ & $\begin{array}{l}\text { Estudio del concepto de in forma ción do cu men taly delos } \\
\text { elemen tos delproceso dela do cu men ta ción. Sis te mas, re des } \\
\text { y centros de información y documentación. }\end{array}$ \\
\hline $\begin{array}{l}\text { Técnicas historiográficas de la in- } \\
\text { vestigación documental } \\
\text { ( } 6 \text { créditos) }\end{array}$ & Paleografía, diplomática y numismática aplicadas \\
\hline $\begin{array}{l}\text { Tecnologías de la información } \\
\text { (15créditos) }\end{array}$ & $\begin{array}{l}\text { Tecnologías de conservación y recuperación de la informa- } \\
\text { ción. Construcción de bases de datosbibliográficas, numéri } \\
\text { cas, textuales y factuales. Edición electrónica. }\end{array}$ \\
\hline $\begin{array}{l}\text { Prácticum } \\
\text { (10créditos) }\end{array}$ & $\begin{array}{l}\text { Conjunto integrado de prácticas de centros universitarioso } \\
\text { vinculados a la universidad por convenios que pon gan a los } \\
\text { estudiantes en contacto con los pro ble mas de la prác ti ca pro- } \\
\text { fesional. }\end{array}$ \\
\hline
\end{tabular}

Los pla nes dees tu diode benserela bo ra dos porla co rrespon dien te JuntadeCentro, que de termina cuáles sonlos progra mas, las ac tivida des, la na tu raleza (en el caso de las asignaturas obligatorias y op ta tivas) y la du ra ción de las ma te rias que se van a cursar, así como su ads crip ción alas di fe ren tes áreas de cono cimien to. Elcontenido específico de las materias definidas es establecidopor los departamentos responsablesdeimpartirlas.

Para obtenerun título los alum nosdeberán supe raruna se rie de cré di tos de ca rácter forzo so y un nú me ro va ria ble de asigna tu ras de ca rác ter li bre. La po si bi li dad de elegiren treunnúmerorelativamenteampliodeasigna turaslespermitealosalumnos la creación de un currículumpersonalizado, en función de sus preferencias yde sus perspectivasprofesionalesylaborales.

Las asignaturas de carácter obligatorio y de carácter voluntario aparecen en los planesdeestudiosclasificadasentresgrandescategorías:

Asignaturas troncales. Constituyen los contenidos mínimos exigibles a un mismo título oficial válido para todo el territorio nacional. Son por tanto obliga to rias en los pla nes de es tu dio. Deben cons ti tuir no menos del $30 \%$ de la car ga do cen te du ran te el pri merci clo, y al me nos el $25 \%$ de la mis ma, en el segundo ciclo. 
Asignaturas no troncales. Se trata de aquellas ma te rias de fini das porcada universidadalaprobarlosplanes de es tudios. Puedenserobliga to riasuoptativas.

* Asignaturas de libre configuración. Materias que el estudiantepuede cursar libre men te yqueincluye su cu rrículum en tre to das las que cada univer sidad oferta con esta categoría.

Si nos cen tra mos en los co mien zos de la for ma ción re gla da en Es pa ña, la pre sencia de la bi blio me tría y de las ma te rias cuan ti ta ti vas ha sido casi nula en los pla nes de estudiosdelasuniversidadesespañolas.

La re forma delos pla nes de es tu diole daba un cier to gra do de au to no mía a las universidades para que in tro du je ran con tenidos propios, aun que nada comparableala que tienenlas universida des eu ropeas y norte ame rica nas. No obs tan te, sepu dieron apre ciarlas apues tas quelos dis tin tos cen tros ha cían y su po sicio na mien to en el campo de las hu ma ni da des o de las cien cias so ciales. La op ción más tra di cio nalerala hur manística, ya que conectaba mejor con el perfil de los profesionales de España. La otra opción elegía un enfoque más tecnológico y las materias cuantitativas; sin embargoparecequenoexisteunaten denciacla ra en ningunsentido.

La estruc tu ra ción delos planes de es tu dio lecon fe ría ala biblio me tríauna ca tegorización dis tin ta en los pla nes de es tu dio en los que te nía pre sen cia, así como en las asignaturas o materiasrelacionadas con ésta, que en cier ta formarecogían procesos cuantitativosensuscontenidos.

Así,lain troduc ción de la biblio me tría yotras ma te rias de cor te cuan tita tivo en los planesdeestudiodelasescuelasy facul tadesdeByDhaseguidounprocesogradual.

Analizaremosacontinuaciónlasmaterias decorte cuantitativoenlosplanes deestudiodelastitulaciones debiblioteconomíaydocumentación.

\section{METOdología}

Seguida mente seex poneel tra tamien to quelas distintasuniversida des le danalos diferentes pla nes de es tu dio vi gen tes en la ac tua li dad so bre biblio me tría y las ma terias relacionadas con ésta, siempre que contenganmétodos cuantitativos. También semencionasudistribucióntomandoencuentalastitulacionescorrespondientesyla categorización deés tas, yse muestra elbalance finaldel procesoderenovación sobre los pla nes de es tu dio de las ma te rias rela cio na das con la cuan ti fica ción delos procesosinformacionales.

Eles tu diose ha es truc tura doen tres gran des blo ques: el primerciclo que otorga el títulode diplo madoen biblio te cono míay do cumentación; el segun doeltítulodelicen cia do en do cu men ta ción; y porúl ti mo el terce ro que adju dica el gra do de doc tor en documentación comoel máximotítuloimpartido en la universidad, quecapacita paralaactividadinvestigadora.

En cada uno de estos bloques se emplea la misma metodología y se aplican los mis mos crite rios. Se ana liza la obliga to rie dad y la op ta tividad en cada uno de los planesdeestudioqueofrecenlasuniversidadesespañolas. 
Seguidamente, antela fal ta denorma liza ción exis ten te enlas dis tin tas deno mina ciones delas asignatu ras, hemosincluidoaquéllas que de al guna maneraes tánvincur ladasalosprocesoscuantitativos delain formación. Noshemoscentradoenéstas.

* Bibliometría

* Informetría

* Estadística

* Estudios de usuarios

* Planificación y evaluación de unidades de información

* Técnicas y métodos aplicados a la investigación en documentación

* Evaluación de la ciencia

Política científica

Ade más sein clui rán el nú me ro de cré di tos, el ca rác ter dela asig na tu ra, el títulode ésta, elcurso en el que seim parte y el añoen que apa re ció pu blica do elPlan dees tu dio en elBOE(BoletínOficialdelEstado).

Acontinuaciónsees tu dia el peso que tienenlas dis tintas ma terias cuantitativas en las es cuelas y facul ta des en rela ción conla carga lec tiva to tal co rres pondien te a cada titu la ción,ysedes glo sanen créditos teó ricosy prác ticos elnú me rode asigna tu ras de carac terobliga to rioy op ta tivo; el peso que tienen las ma te rias cuan tita tivas de carácterobliga to rio (asigna tu ras tron ca les yobliga to rias) en rela ción conel to tal de créditos obligatorios; y el peso de las asignaturas optativas de corte cuantitativo en relación con el total de créditos optativos para cada titulación. También se compara la situa ciónactual deca ráctercuan tita tivoy la rela ción dees tos créditosconla carga lectiva to tal para cada titula ción en las di feren tes univer sida des queimpartenalguna titulacióndeByDenespaña.

\section{RESULTADOS}

Los resultados obtenidos son los que aparecen reflejados en los tres apartados siguientes, ysecorrespondenconlostresbloquesenquees tructuramosnuestrotrabajo.

\section{Diplomatura}

En la tabla 2 aparecen re cogidaslasasignatu ras de cortecuan tita tivoenladiploma tura y se ob serva que el pro me dio de asig na tu ras de cor te cuan ti ta tivo es de 1,3 asignatu ras poruniversidad.Laque más asigna tu ras presenta es laUniversidad deEx tremadura y la que menos la Universidad de León, que muestra una clara tendencia hu manís tica en suplan de es tu dios.Labibliometría como asigna tura aparece bajoesa denominación en seis universidades (Complutense, Granada, Zaragoza, Extremadu ra, Valen ciay La Coruña) delastrecequeimpartenla diplo ma tura; en to das ellasla bibliome tría tienecarácterdeop ta tivamenosen GranadayEx tre ma du radondeapa rece como obliga to ria. Nada de esto es ex traño si te ne mos en cuen ta que en la co misión creada ad hoc, para elaborar el Plan de estudios de la diplomatura, participaron 
profesores delaUniversidadde Granada, quienesdejaronsentirsutendencia porlos estudioscuantitativos.

Tabla 2 Distribución de asignaturas decortecuantitativoenladiplomatura

\begin{tabular}{|c|c|c|c|c|c|}
\hline Universidad & Carga & Asignaturas & Créditos & Tipo & Curso \\
\hline $\begin{array}{l}\text { Carlos III } \\
\text { Plan } 1994 \\
\end{array}$ & 202 & Es tu dios de usua rios & 7 & $\mathrm{O}$ & $3^{\circ}$ \\
\hline \multirow{2}{*}{$\begin{array}{l}\text { Complutense } \\
\text { Plan } 2000\end{array}$} & \multirow{2}{*}{202,5} & $\begin{array}{l}\text { Estadística, bibliometría e } \\
\text { informetría. }\end{array}$ & 4,5 & Op & \\
\hline & & Es tu dios y For ma ción de usua rios & 6 & Op & \\
\hline $\begin{array}{l}\text { León } \\
\text { Plan } 1995 \\
\end{array}$ & 206 & & & & \\
\hline \multirow{2}{*}{$\begin{array}{l}\text { Granada } \\
\text { Plan } 2001\end{array}$} & \multirow[t]{2}{*}{206} & $\begin{array}{l}\text { Introducción a la estadística y la } \\
\text { bibliometría }\end{array}$ & 8 & $\mathrm{O}$ & $2^{\circ}$ \\
\hline & & Es tu dios de usua rios y nec. de inf. & 6 & $\mathrm{O}$ & $2^{\circ}$ \\
\hline \multirow{2}{*}{$\begin{array}{l}\text { Murcia } \\
\text { Plan } 2000\end{array}$} & \multirow{2}{*}{188} & Estadísticabásica en doc. & 5 & Op & \\
\hline & & Técnicas de inv. científica & 5 & Op & \\
\hline $\begin{array}{l}\text { Salamanca } \\
\text { Plan } 2000\end{array}$ & 188 & Evaluación de fuentes de inf. & 4,5 & Op & \\
\hline $\begin{array}{l}\text { Zaragoza } \\
\text { Plan } 1996 \\
\end{array}$ & 205 & Informetríaybibliometría & 6 & Op & \\
\hline $\begin{array}{l}\text { La Coruña } \\
\text { Plan } 1997 \\
\end{array}$ & 202 & Bibliometría y estadística & 6 & Op & \\
\hline \multirow{2}{*}{$\begin{array}{l}\text { Barcelona } \\
\text { Plan } 1999 \\
\end{array}$} & \multirow{2}{*}{186} & Estadísticaaplicada & 4,5 & Op & \\
\hline & & Es tu dios y for ma ción de usua rios & 4,5 & Op & \\
\hline \multirow{2}{*}{$\begin{array}{l}\text { Valencia } \\
\text { Plan } 2000\end{array}$} & \multirow{2}{*}{180} & Estadística & 6 & Op & \\
\hline & & Introducción a la bibliometría & 4,5 & Op & \\
\hline $\begin{array}{l}\text { Vic } \\
\text { Plan } 2001 \\
\end{array}$ & 194 & Métodos estadísticos en ByD & 6 & Op & \\
\hline \multirow{3}{*}{$\begin{array}{l}\text { Extremadura } \\
\text { Plan } 1995\end{array}$} & \multirow{3}{*}{189} & Fundamentosdebibliometría & 6 & $\mathrm{O}$ & $1^{\mathrm{o}}$ \\
\hline & & Estadística & 6 & Op & \\
\hline & & Es tu dios de usuarios & 6 & Op & \\
\hline
\end{tabular}


En la ta bla 3 sere cogen desglo sa das la obliga to rie dadylaop ta tividad, el nú me ro de cré di tos teó ricos y prác ticos, y el peso que tie nen las asigna tu ras de cor te cuan titativo con la cargalec tiva obliga to ria y op ta tiva. Parala obliga to rie dad en diplomatura (los porcen tajes sehan cal cula do a partir del núme ro decré di tos de carác tercuan titativo en relación con el núme ro de crédi tos obliga to rios, tron cales yobliga to rios) se observa que sólo tres universidades presentan materias cuantitativas (Carlos III, GranadayEx tremadura); deellas, CarlosIIIy Extremaduranopresentandiferencias significativas 4,93\% y 5,22\% respectivamente; no así Granada que las duplica con 10,73\%delacargalectivaobligatoria.

Tabla 3 Distribución de créditos teórico-prácticos con carácter obligatorio u optativo en la diplomatura

\begin{tabular}{|c|c|c|c|c|c|c|c|c|c|c|c|}
\hline \multicolumn{9}{|c|}{ OBLIGATORIEDAD } & \multicolumn{5}{c|}{ OPTATIVIDAD } \\
\hline Universidad & Teóricos & Prácticos & Totales & $\begin{array}{c}\text { Obligatoriedad } \\
\%\end{array}$ & $\begin{array}{c}\mathbf{N}^{\circ} \\
\text { Asig. }\end{array}$ & Teóricos & Prácticos & Totales & $\begin{array}{c}\text { Opta tivi dad } \\
\%\end{array}$ & $\begin{array}{c}\mathbf{N}^{\circ} \\
\text { Asig. }\end{array}$ & $\begin{array}{c}\text { Carga } \\
\text { lectiva }\end{array}$ \\
\hline Barcelona & & & 0 & 0,00 & 0 & & & 9 & 9,38 & 2 & 4,84 \\
\hline Carlos III & 4 & 3 & 7 & 4,93 & 1 & & & 0 & 0,00 & 0 & 3,47 \\
\hline CEU & & & 0 & 0,00 & 0 & 0 & 0 & 0 & 0,00 & 0 & 0,00 \\
\hline Complutense & & & 0 & 0,00 & 0 & 4 & 6,5 & 10,5 & 5,80 & 2 & 5,19 \\
\hline Extremadura & 3 & 3 & 6 & 5,22 & 1 & 6 & 6 & 12 & 11,11 & 2 & 9,52 \\
\hline Granada & 7 & 7 & 14 & 10,73 & 2 & & & 0 & 0,00 & 0 & 7,51 \\
\hline LaCoruña & & & 0 & 0,00 & 0 & 4 & 2 & 6 & 11,11 & 1 & 2,93 \\
\hline León & & & 0 & 0,00 & 0 & & & 0 & 0,00 & 0 & 0,00 \\
\hline Murcia & & & 0 & 0,00 & 0 & 6 & 4 & 10 & 9,09 & 2 & 5,32 \\
\hline Salamanca & & & & 0,00 & & 1 & 3,5 & 4,5 & 4,00 & 1 & 2,39 \\
\hline Valencia & & & 0 & 0,00 & 0 & 9 & 4,5 & 13,5 & 11,89 & 3 & 7,50 \\
\hline Vic & & & 0 & 0,00 & 0 & 3 & 3 & 6 & 5,56 & 1 & 3,09 \\
\hline Zaragoza & & & 0 & 0,00 & 0 & 4 & 2 & 6 & 4,44 & 1 & 2,93 \\
\hline
\end{tabular}

Encuan to ala op ta tividad seob tiene que el ma yor porcen taje de créditos seda en laUniversidadde Valen cia, con 11,89\% dela cargalec tivaopta tiva, seguidapor Gra nada y Extremadura, con un 11,11 \%, mien trasque el nú me rode universida desque notienen asigna tu rasop tativas decortecuantita tivo se hareducidoatres (León, C arlos III y CEU), tal y como se pue de ob ser varen la figu ra 1. (Para su cál cu lo se ha con sideradoelnúmerode créditos delasasigna tu rasop ta tivas decarác tercuan tita tivo en relaciónconeltotaldecréditosdeasignaturasoptativas).

En la figura 2 se refleja el peso de las materias cuantitativas (tanto obligatorias como op ta tivas) con rela ción a la carga lec tiva de la di plo ma tu ra, y se ob serva quela uni versi dad que más peso tie ne es la de Ex tre ma du ra, con $9,52 \%$ delos cré ditos, seguidaporValen cia, Granada, MurciaylaCompluten se. Las demás seen cuentranentreel2\%y $5 \%$ delacargasalvoLeón, quenotienepeso alguno. 
Figura 1. Distribución de créditosobligatorios y optativos

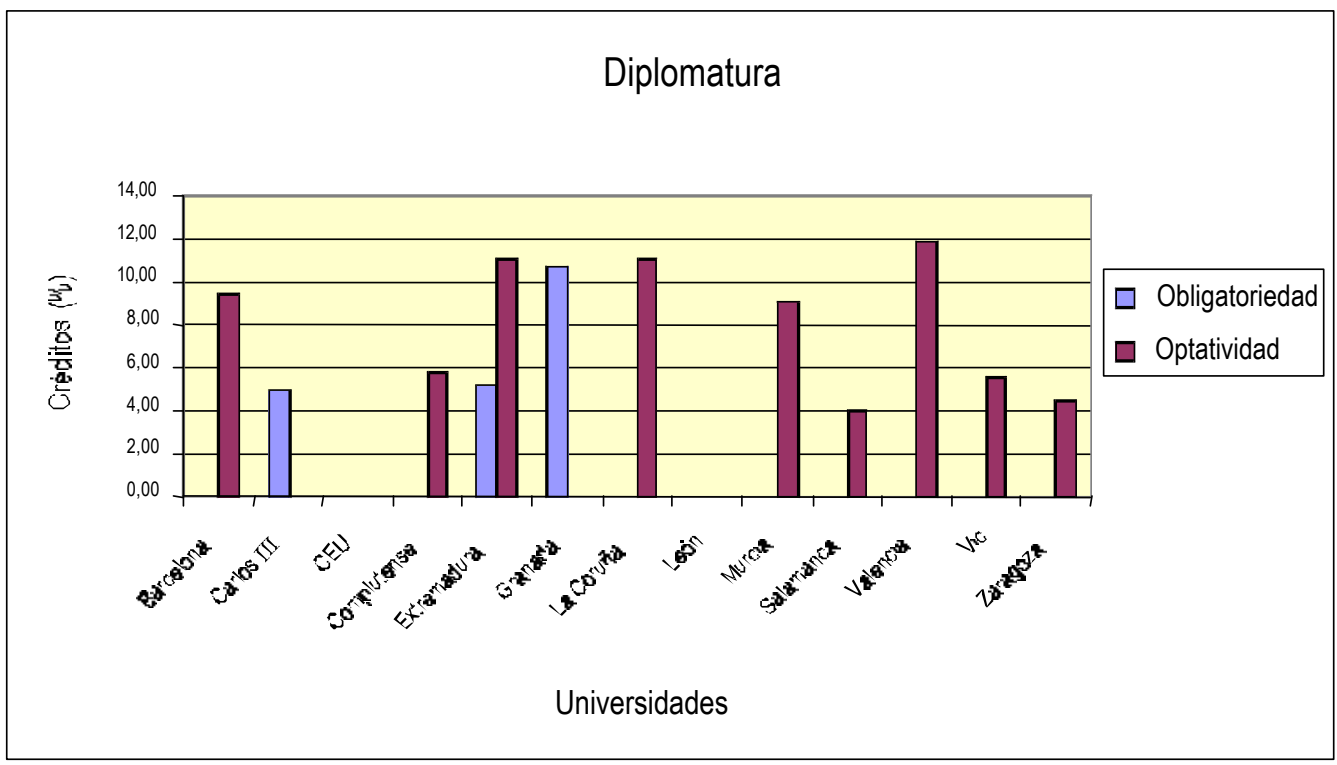

Figu ra 2. Dis tri bu ción de crédi tos con re la ción a la carga lectiva

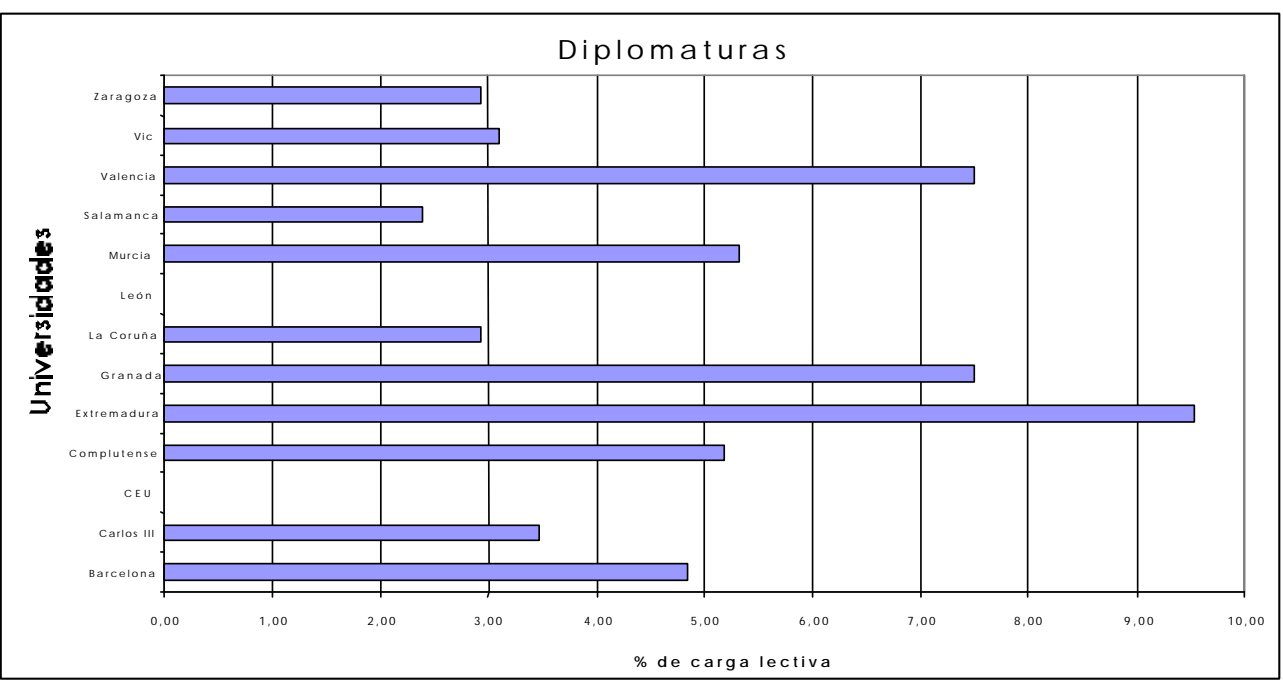




\section{LICENCIATURA}

Enlalicencia turahemos seguido la mis maes truc turaqueenla diploma tura. Enla tabla4serecogenlasuniversidades, planes deestudio, cargalec tiva, asignaturas, créditos, tipoycurso, yhemos ob tenidoun promediode asignatu ras de cortecuan tita tivo de 3,8 asig na tu ras poruni ver sidad. Es la Univer si dad de Al ca lála que más asig na tu ras im par te con un to tal de cin co, y la que me nos lo hace es la Univer si dad Ober ta de Ca ta lu ña con un to tal de dos. Con re la ción a la di plo ma tu ra se pro du ce un in crementode2,5asignaturasporuniversidadenlalicenciatura.

La asignatura de bibliometría aparece como tal en las siguientes universidades: Carlos III,Alcalá,Salamanca,Barcelona, Politécnicade Valencia, ylain formetría se impartesolamenteenlaUniversidaddeExtremadura.

Tabla 4.Dis tribucióndeasignaturas decortecuantitativoen licenciatura

\begin{tabular}{|c|c|c|c|c|c|}
\hline Universidad & carga & Asignaturas & Créditos & Tipo & Curso \\
\hline \multirow{4}{*}{$\begin{array}{l}\text { Carlos III } \\
\text { Plan } 1994\end{array}$} & \multirow{4}{*}{140} & Métodos estadísticos & 7 & $\mathrm{~T}$ & $1^{\mathrm{o}}$ \\
\hline & & Evaluación de serv. y form. de us. & 6 & $\mathrm{O}$ & $1^{\mathrm{o}}$ \\
\hline & & Bibliometría & 6 & $\mathrm{~T}$ & $2^{\circ}$ \\
\hline & & Técn. de ev., control de und. de inf. & 7 & $\mathrm{~T}$ & $2^{\mathrm{o}}$ \\
\hline \multirow{3}{*}{$\begin{array}{l}\text { Complutense } \\
\text { Plan } 1996\end{array}$} & \multirow{3}{*}{137} & Planif. y ev. de sist. de inf. y doc. & 6 & $\mathrm{~T}$ & $1^{\mathrm{o}}$ \\
\hline & & Técnicas de inv. científica & 3 & $\mathrm{~T}$ & $1^{\mathrm{o}}$ \\
\hline & & Estadística aplica da a doc. & 6 & $\mathrm{~T}$ & $2^{\circ}$ \\
\hline \multirow{6}{*}{$\begin{array}{l}\text { Alcalá } \\
\text { Plan } 1995\end{array}$} & \multirow{6}{*}{133,5} & Planif. y ev. de sist. de inf. & 6 & $\mathrm{~T}$ & $1^{\circ}$ \\
\hline & & Evaluación de serv. y form. de us. & 6 & $\mathrm{O}$ & $1^{\circ}$ \\
\hline & & $\begin{array}{l}\text { Técnicas documentalesaplicadasa } \\
\text { la doc. }\end{array}$ & 9 & $\mathrm{~T}$ & $2^{\circ}$ \\
\hline & & Bibliometría & 6 & $\mathrm{O}$ & $2^{\circ}$ \\
\hline & & Estadística & 9 & $\mathrm{~T}$ & $1^{\mathrm{o}}$ \\
\hline & & $\begin{array}{l}\text { Gestión y ev. de catálogosautoma } \\
\text { tizados }\end{array}$ & 6 & $\mathrm{OP}$ & \\
\hline \multirow{4}{*}{$\begin{array}{l}\text { Granada } \\
\text { Plan } 2001\end{array}$} & \multirow{4}{*}{120} & Estadística & 6 & $\mathrm{~T}$ & $1^{\mathrm{o}}$ \\
\hline & & $\begin{array}{l}\text { Técnicas documentalesaplicadasa } \\
\text { la inv. }\end{array}$ & 6 & $\mathrm{~T}$ & $1^{\mathrm{o}}$ \\
\hline & & Planif. y ev. de sist. de inf. y doc. & 6 & $\mathrm{~T}$ & $2^{\circ}$ \\
\hline & & $\begin{array}{l}\text { Ev. científica y fuentes de in forma } \\
\text { ción especializada }\end{array}$ & 11 & $\mathrm{OP}$ & \\
\hline
\end{tabular}


Tabla 4.Distribución deasignaturas decortecuantitativoen licenciatura (Cont.)

\begin{tabular}{|c|c|c|c|c|c|}
\hline Universidad & carga & Asignaturas & Créditos & Tipo & Curso \\
\hline \multirow{3}{*}{$\begin{array}{l}\text { Murcia } \\
\text { Plan } 2000\end{array}$} & \multirow{3}{*}{137} & Estadística & 6 & $\mathrm{~T}$ & $1^{\circ}$ \\
\hline & & Técnicasaplicadas a la inv. & 6 & $\mathrm{~T}$ & $1^{\circ}$ \\
\hline & & Pla nif. y ev. de sist. de inf. y doc. & 6 & $\mathrm{~T}$ & $1^{\circ}$ \\
\hline \multirow{5}{*}{$\begin{array}{l}\text { Salamanca } \\
\text { Plan } 2000\end{array}$} & \multirow{5}{*}{120} & Pla nif. y ev. de uni da des de inf. & 7 & $\mathrm{~T}$ & $1^{\circ}$ \\
\hline & & $\begin{array}{l}\text { Técnicascuantitativas de inv. en } \\
\text { doc. }\end{array}$ & 7 & $\mathrm{~T}$ & $1^{\circ}$ \\
\hline & & Estadística aplicada a la c. de la doc. & 7 & $\mathrm{~T}$ & $1^{\circ}$ \\
\hline & & Bibliometría & 4,5 & OP & \\
\hline & & Evaluación de la ciencia & 4,5 & OP & \\
\hline \multirow{3}{*}{$\begin{array}{l}\text { Autónoma de } \\
\text { Barcelona } \\
\text { Plan } 2000\end{array}$} & \multirow{3}{*}{123,5} & Pla nif. y ev. de sist. de inf. & 6 & $\mathrm{O}$ & $1^{\circ}$ \\
\hline & & $\begin{array}{l}\text { Técnicasdocumentales aplicadasa } \\
\text { la inv. }\end{array}$ & 6 & $\mathrm{O}$ & $1^{\circ}$ \\
\hline & & Estadística & 6 & $\mathrm{O}$ & $1^{\circ}$ \\
\hline \multirow{3}{*}{$\begin{array}{l}\text { Barcelona } \\
\text { Plan } 1999\end{array}$} & \multirow{3}{*}{120} & Estadística aplicada a la doc. & 6 & $\mathrm{~T}$ & $1^{\circ}$ \\
\hline & & Pla nif. y ev. de sist. de inf. y doc. & 6 & $\mathrm{~T}$ & $1^{\circ}$ \\
\hline & & Técnicas est. ybiblio métricas & 6 & OP & \\
\hline \multirow{2}{*}{$\begin{array}{l}\text { Oberta de Cata- } \\
\text { luña } \\
\text { Plan } 1999 \\
\end{array}$} & \multirow[b]{2}{*}{120} & Estadística & 6 & $\mathrm{~T}$ & $1^{\mathrm{o}}$ \\
\hline & & $\begin{array}{l}\text { Técnicasdocumentales aplicadasa } \\
\text { la inv. }\end{array}$ & 6 & $\mathrm{~T}$ & $2^{\circ}$ \\
\hline \multirow{4}{*}{$\begin{array}{l}\text { Politécnica de } \\
\text { Valencia } \\
\text { Plan } 1996\end{array}$} & & $\begin{array}{l}\text { Técnicasdocumentales aplicadasa } \\
\text { la inv. }\end{array}$ & 6 & $\mathrm{~T}$ & $2^{\circ}$ \\
\hline & & Bibliometría & 6 & OP & \\
\hline & & Estadística & 6 & $\mathrm{~T}$ & $1^{\circ}$ \\
\hline & & Pla nif. y ev. de sist. de inf. & 6 & $\mathrm{~T}$ & $2^{\circ}$ \\
\hline \multirow{5}{*}{$\begin{array}{l}\text { Extremadura } \\
\text { Plan } 1998\end{array}$} & & Estadística & 6 & $\mathrm{~T}$ & \\
\hline & & Informetría & 6 & $\mathrm{O}$ & $1^{\mathrm{o}}$ \\
\hline & & Pla nif. y ev. de uni da des de inf. & 6 & $\mathrm{~T}$ & $1^{\circ}$ \\
\hline & & Téc ni cas doc. apli ca das a la inv. & 6 & $\mathrm{~T}$ & $2^{\circ}$ \\
\hline & & Política científica & 6 & OP & $2^{\circ}$ \\
\hline
\end{tabular}

$$
\begin{aligned}
& T=\text { Troncal } \\
& O=\text { Obligatoria } \\
& O p=\text { Optativa }
\end{aligned}
$$


Con rela ción al peso delas asigna tu ras de cor te cuan ti ta tivo en licen cia tu ra (tabla 5) con base en la obligatoriedad y optatividad, observamos que la universidad que presentamayorporcentajedeasignaturasobligatoriases Salamanca, con un $38,86 \%$, segui da de Ex tre ma du ra $(38,10)$, Alcalá $(36,92)$ y Murcia con el $34,48 \%$ (figura3). Se produceunincrementomuysignificativoconrelaciónala diplomatura, dondeelmayorpesodelasasignaturas recaíaenlaoptatividad.

Tabla 5

Distribucióndecréditosteórico-prácticosconcarácterobligatorio u optativoenlalicenciatura

\begin{tabular}{|c|c|c|c|c|c|c|c|c|c|c|c|}
\hline \multicolumn{6}{|c|}{ OBLIGATORIEDAD } & \multicolumn{6}{|c|}{ OPTATIVIDAD } \\
\hline Universidad & Teóricos & Prácticos & Totales & $\begin{array}{c}\text { Obligatoriedad } \\
\% \\
\end{array}$ & $\begin{array}{l}\mathbf{N}^{\circ} \\
\text { Asig. }\end{array}$ & Teó ricos & Prácticos & Totales & $\begin{array}{c}\text { Optatividad } \\
\% \\
\end{array}$ & $\begin{array}{l}\mathrm{N}^{\circ} \\
\text { Asig. }\end{array}$ & $\begin{array}{c}\text { Car ga lec- } \\
\text { tiva } \%\end{array}$ \\
\hline Al ca lá de $\mathrm{H}$. & 22,5 & 13,5 & 36 & 36,92 & 5 & 2 & 4 & 6 & 11,43 & 1 & 31,46 \\
\hline $\begin{array}{c}\text { Autónomade } \\
\text { Barcelona }\end{array}$ & 9 & 7,5 & 16,5 & 17,74 & 3 & & & 0 & 0,00 & 0 & 13,36 \\
\hline Barcelona & 8 & 4 & 12 & 16,67 & 2 & & & 6 & 6,25 & 1 & 15,00 \\
\hline Car los III & 14 & 11 & 25 & 24,51 & 4 & & & 0 & 0,00 & 0 & 17,86 \\
\hline Complutense & 9 & 6 & 15 & 21,13 & 3 & & & 0 & 0,00 & 0 & 10,95 \\
\hline Extremadura & 13 & 11 & 24 & 38,10 & 4 & 3 & 3 & 6 & 4,76 & 1 & 23,08 \\
\hline Granada & 12 & 6 & 18 & 26,87 & 3 & 5 & 6 & 11 & 15,49 & 1 & 19,17 \\
\hline Murcia & 14 & 6 & 20 & 34,48 & 3 & & & 0 & 0,00 & 0 & 11,38 \\
\hline Polit.Valencia & 9 & 9 & 18 & 21,43 & 3 & 3 & 3 & 6 & 5,56 & 1 & 17,78 \\
\hline Salamanca & 14 & 7 & 21 & 38,89 & 3 & 6 & 3 & 9 & 8,00 & 2 & 25,00 \\
\hline UOC & 8 & 4 & 12 & 15,69 & 2 & & & 0 & 0,00 & 0 & 10,00 \\
\hline
\end{tabular}

Laoptatividadenlalicencia turapresentamenospesoquela obliga to riedad, mientrasqueenladiplomaturapresentamáspesola optatividadquela obligato riedad.

Encuantoalcompu to to talde créditos deasigna tu ras decor te cuan tita tivo, conrelaciónala cargalectiva total(figura 4), ob servamosquelas universidadesenlas que más peso tienenés tos son Alcalá (31,4\%), Sala manca (25\%), Ex tre ma du ra (23,07\%), Gra nada $(19,16 \%)$,yla quemenos tieneeslaUOCconun $10 \%$ delacargalectivatotal. 


\section{Figura3}

Distribución de créditos obligatorios y optativos

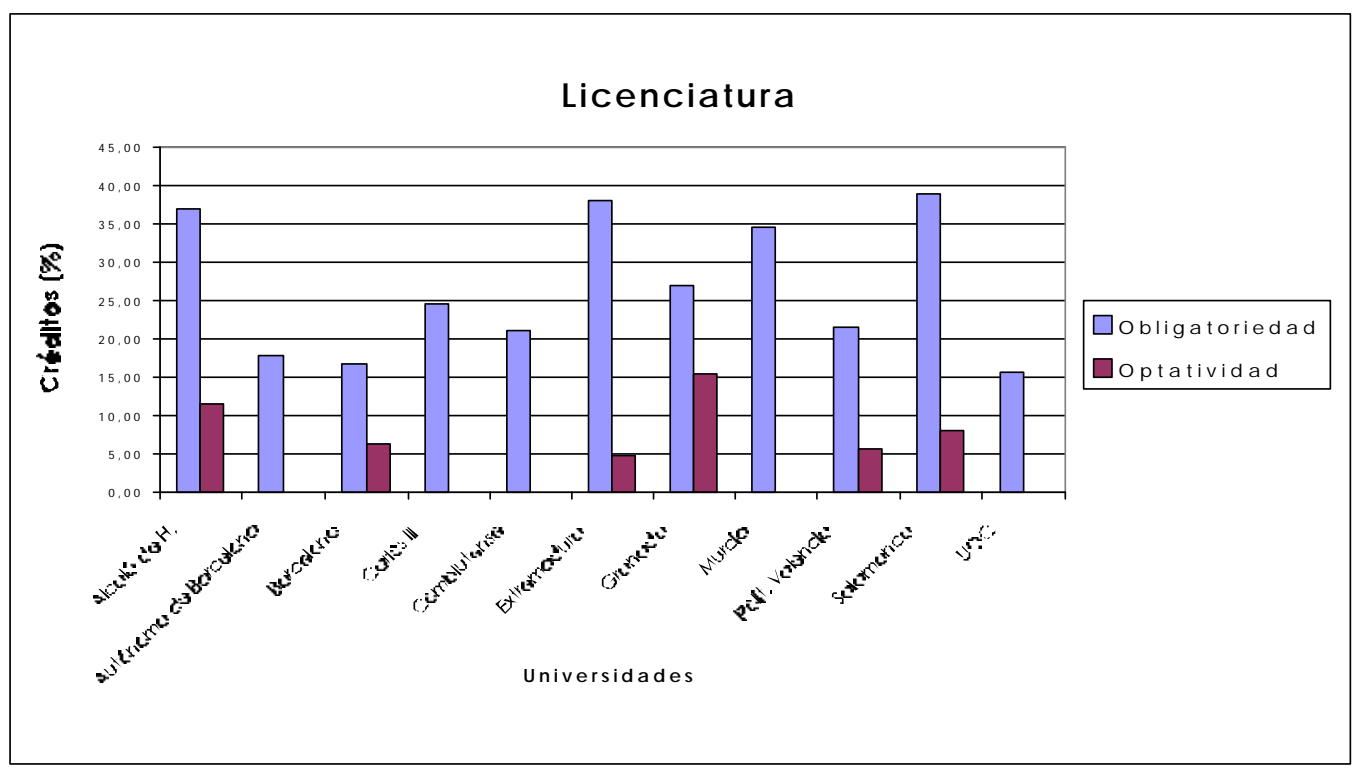

Figura 4

Distribución de créditos con relación a la cargalectiva

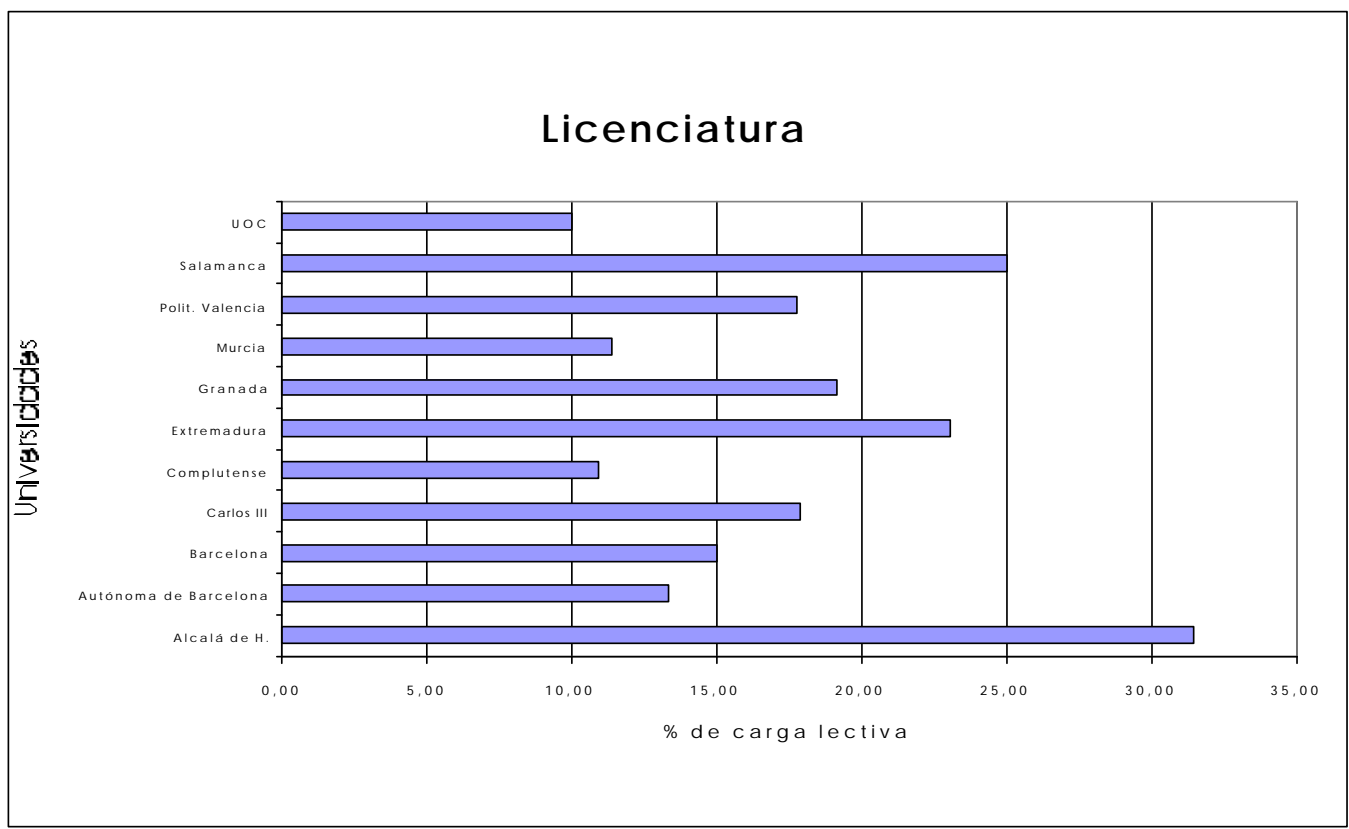




\section{DOCTORADO}

En la tabla 6 se han re co gido los nom bres delos cur sosim partidos en tercerciclo, además delos créditos de cadauno deellos.

\section{Tabla 6}

Distribución de cursos de doctorado de corte cuantitativo

\begin{tabular}{|c|c|c|c|c|}
\hline Universidad & Carga & Cursos & Créditos & Programa \\
\hline Alcalá (1998) & 32 & & & $\begin{array}{l}\text { Inf. doc. y com. } \\
\text { (Dpto de CC de la } \\
\text { computación) }\end{array}$ \\
\hline \multirow{3}{*}{$\begin{array}{l}\text { Barcelona } \\
(2001 / 2003)\end{array}$} & \multirow{3}{*}{32} & $\begin{array}{l}\text { Análisis de citas en la gestión de } \\
\text { las col. }\end{array}$ & 3 & \multirow{3}{*}{$\begin{array}{l}\text { Doc. y sist. docu- } \\
\text { mentales en el en tor- } \\
\text { no digital } \\
\text { (Dpto ByD) }\end{array}$} \\
\hline & & $\begin{array}{l}\text { Técnicas multivariantes aplica das a } \\
\text { la doc. }\end{array}$ & 3 & \\
\hline & & Estudios bibliométricos & 6 & \\
\hline \multirow{3}{*}{ Carlos III } & \multirow{3}{*}{32} & $\begin{array}{l}\text { Estudios de consumo de inf. cien- } \\
\text { tífica }\end{array}$ & 3 & \multirow{3}{*}{ Documentación } \\
\hline & & Indicadores para evaluar la Cy $\mathrm{T}$ & 3 & \\
\hline & & Evaluación de rev. científicas & 2 & \\
\hline $\begin{array}{l}\text { Complutense } \\
(2001 / 2002)\end{array}$ & 32 & & & $\begin{array}{l}\text { Documentación: } \\
\text { fun da men tos y tec- } \\
\text { nología } \\
\text { (Dpto ByD) } \\
\end{array}$ \\
\hline \multirow[t]{3}{*}{$\begin{array}{l}\text { Granada } \\
(2001 / 2002)\end{array}$} & 32 & $\begin{array}{l}\text { Fundamentos de clasificaciónes- } \\
\text { tadística de inf. cient. } \\
\text { La evalua ción de la cien cia: as pec- } \\
\text { tos metodológicos } \\
\text { Las revistas científicas como ins- } \\
\text { tru men tos de trans fe ren cia deinf. } \\
\text { Metodologíapara la ev. de las co- } \\
\text { lecciones de una biblioteca }\end{array}$ & $\begin{array}{l}4 \\
3 \\
3 \\
3\end{array}$ & \multirow[t]{3}{*}{$\begin{array}{l}\text { Documentación e in- } \\
\text { formación científica } \\
\text { (Dpto de ByD) }\end{array}$} \\
\hline & & $\begin{array}{l}\text { Métodos de inferencia y predic- } \\
\text { ción en procesos de análisis de inf. } \\
\text { científica }\end{array}$ & 3 & \\
\hline & & $\begin{array}{l}\text { Técnicas de visualización para el } \\
\text { análisis de la inf. cient. }\end{array}$ & 3 & \\
\hline
\end{tabular}




\section{Tabla 6}

Distribución de cursosdedoctoradodecortecuantitativo (Cont.)

\begin{tabular}{|c|c|c|c|c|}
\hline $\begin{array}{l}\text { Universi- } \\
\text { dad }\end{array}$ & Carga & Cursos & Créditos & Programa \\
\hline $\begin{array}{l}\text { Polit. } \\
\text { Valencia } \\
\text { 2001/2002 }\end{array}$ & 32 & $\begin{array}{l}\text { Indicadores de actividad científica y } \\
\text { modelosbibliométricos } \\
\text { Metodología de la inv. científica } \\
\text { Métodos cuantitativosaplicados a } \\
\text { la doc.: modelos informétricos }\end{array}$ & $\begin{array}{l}3 \\
3 \\
3\end{array}$ & $\begin{array}{l}\text { Ges tión de inf. } \\
\text { (Dpto. de com. au- } \\
\text { diov, doc. e h. del } \\
\text { arte) }\end{array}$ \\
\hline \multirow{4}{*}{$\begin{array}{l}\text { Salamanca } \\
(2000 / 2002)\end{array}$} & \multirow{4}{*}{32} & $\begin{array}{l}\text { Fundamentos y aplicaciones de los } \\
\text { estudios cuantitativos so bre la cien- } \\
\text { cia }\end{array}$ & 3 & \multirow{4}{*}{$\begin{array}{l}\text { Metodologías y líneas } \\
\text { de investigación en } \\
\text { ByD } \\
\text { (Dpto. ByD) }\end{array}$} \\
\hline & & $\begin{array}{l}\text { Cuantificación, evaluación y toma } \\
\text { de decisiones en uni da des deinf. }\end{array}$ & 3 & \\
\hline & & $\begin{array}{l}\text { La evaluación científica de institut } \\
\text { ciones median teméto dos biblio mé } \\
\text { tricos }\end{array}$ & 3 & \\
\hline & & $\begin{array}{l}\text { Metodología estadísticapara inves- } \\
\text { tigación }\end{array}$ & 5 & \\
\hline $\begin{array}{l}\text { Valencia } \\
(2001 / 2002)\end{array}$ & 32 & Indicadores de actividad científica & 3 & $\begin{array}{l}\text { Documentación } \\
\text { (Dpto. } \mathrm{H}^{\mathrm{o}} \text { de la cien- } \\
\text { cia y doc.) }\end{array}$ \\
\hline Zaragoza & 32 & & & $\begin{array}{l}\text { Sistemas de inf. y doc. } \\
\text { (Dpto. CC de doc. e } \\
\mathrm{H}^{\circ} \text { de la c.) }\end{array}$ \\
\hline $\begin{array}{l}\text { Oberta de } \\
\text { Cataluña } \\
(2001 / 2003) \\
\end{array}$ & 32 & $\begin{array}{l}\text { Métodos cuantitativospara la in- } \\
\text { vestigación sobre la sociedad de la } \\
\text { inf. }\end{array}$ & 2,5 & $\begin{array}{l}\text { So cie dad de la in for- } \\
\text { mación y el conoci- } \\
\text { miento }\end{array}$ \\
\hline \multirow{2}{*}{$\begin{array}{l}\text { Extremadura } \\
2001 / 2002\end{array}$} & \multirow{2}{*}{32} & $\begin{array}{l}\text { Aproximación a algunasinvestiga } \\
\text { cionesmetodológicas en documen- } \\
\text { tación }\end{array}$ & 2 & \multirow{2}{*}{$\begin{array}{l}\text { Comunicación y docu- } \\
\text { mentación } \\
\text { (Dpto. informática) }\end{array}$} \\
\hline & & $\begin{array}{l}\text { Técnicas informétricas multivarian- } \\
\text { tes apli ca das al análi sis de la do cu- } \\
\text { mentación científica }\end{array}$ & 3 & \\
\hline
\end{tabular}

En la tabla 7 sehareflejadoelporcen taje créditos de cor tecuan tita tivo en rela ción conlacargalectiva deldoctorado, dondese observaqueSala mancaha op tadoporun claro posicionamiento por las materias de carácter cuantitativo en la licenciatura y doc to ra do, y que está a la ca be za con 34,5\% y es se guida por Grana da con un 32,76\%. Existen tres universidades en las que no aparece ningún curso en el que tengan 
presencialos métodoscuantitativos, Alcalá,Leóny Complutense(figura 5), aunque hay que recordar que Al calá es la que pre sen ta ma yorcarga en la licen cia tu ra en este tipodeasignaturas.

Tabla 7

Distribucióndecréditoscuantitativosendoctorado

\begin{tabular}{|l|c|c|c|}
\hline \multicolumn{1}{|c|}{ Universidad } & Créditos & $\boldsymbol{\%}$ & $\mathbf{N}^{\circ}$ CURSOS \\
\hline Alcalá de H. & 0 & 0,00 & 0 \\
\hline Barcelona & 12 & 19,05 & 3 \\
\hline Carlos III & 8 & 12,70 & 3 \\
\hline Complutense & 0 & 0,00 & 0 \\
\hline Extremadura & 5 & 9,26 & 2 \\
\hline Granada & 19 & 32,76 & 6 \\
\hline León & 0 & 0,00 & 0 \\
\hline Murcia & 6 & 12,50 & 2 \\
\hline Polit. Valencia & 9 & 13,04 & 3 \\
\hline Salamanca & 14 & 34,15 & 4 \\
\hline UOC & 2,5 & 4,27 & 1 \\
\hline Valencia & 3 & 8,33 & 1 \\
\hline Zaragoza & 4 & 6,78 & 1 \\
\hline
\end{tabular}

Figura 5

Distribución de créditos en doctorado

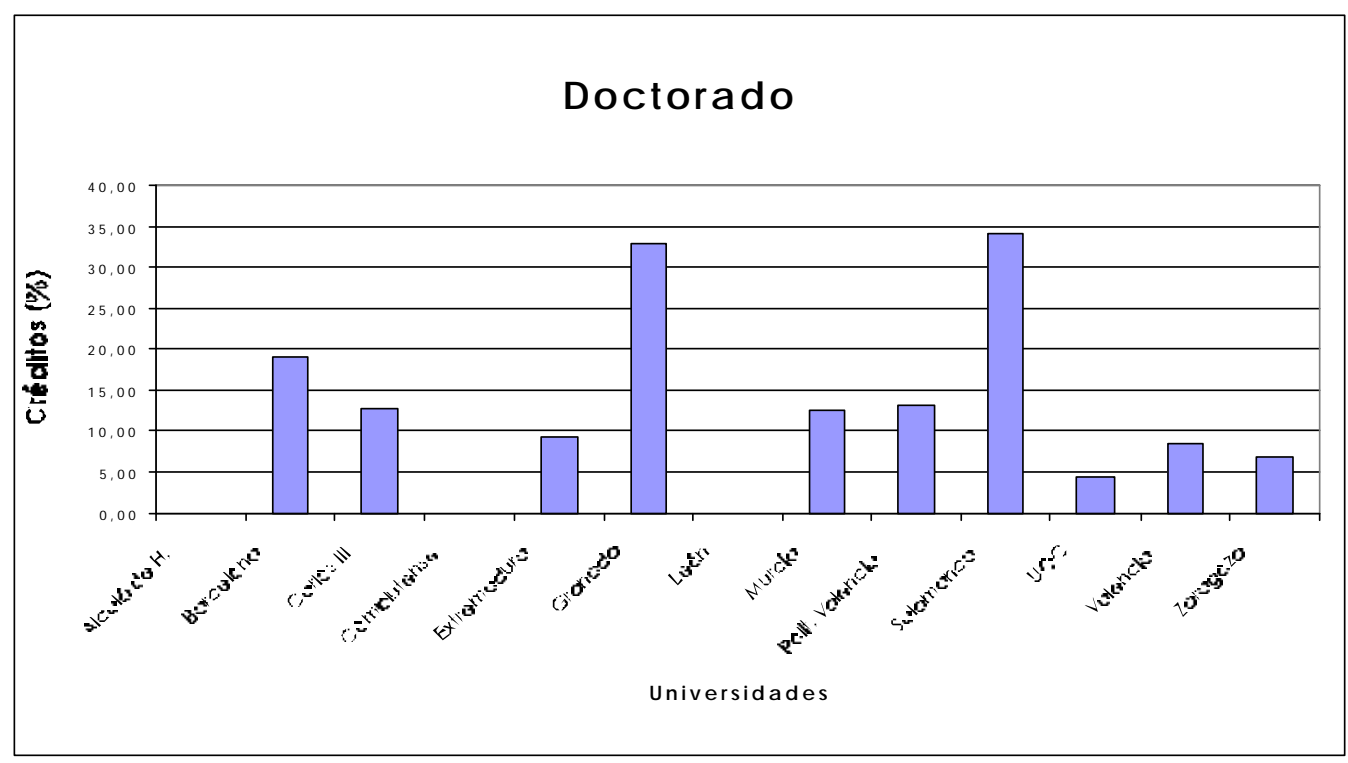


Para finalizarnues tro es tu dio he mos creído convenien te re flejaren la tabla 8 una comparativadelasitua ciónac tualen las escuelas y fa cul ta des de ByD sobrelas materias de cortecuan ti ta tivo ex presadasen cré di tos y su rela ción conla cargalec tiva total. Intentamos reflejar y constatar con mayor claridad las tendencias de formación en España entre la diplomatura, la licenciatura y el doctorado. Por ejemplo, la Universidad de Granadapasódel 6,8\% enladiploma turayconunposicionamien to cla ro con relación alas materias cuan ti ta tivas, a casitriplicarse en licen cia tu ray cuadruplicarseendoctorado.

\section{Tabla 8}

Comparativa de créditoscuantitativos en las tres titulaciones

\begin{tabular}{|c|c|c|c|}
\hline Universidad & Diplomatura & Licenciatura & Doctorado \\
\hline Barcelona & $\begin{array}{c}9 \\
(4,84) \\
\end{array}$ & $\begin{array}{r}18 \\
(15)\end{array}$ & $\begin{array}{c}12 \\
(19,05 \\
\end{array}$ \\
\hline Carlos III & $\begin{array}{c}7 \\
(3,47) \\
\end{array}$ & $\begin{array}{c}25 \\
(17,86) \\
\end{array}$ & $\begin{array}{c}8 \\
(12,70) \\
\end{array}$ \\
\hline $\mathrm{CEU}$ & 0 & & \\
\hline Complutense & $\begin{array}{c}10,5 \\
(5,19) \\
\end{array}$ & $\begin{array}{c}15 \\
(10,95) \\
\end{array}$ & $\begin{array}{c}0 \\
(0) \\
\end{array}$ \\
\hline Extremadura & $\begin{array}{c}18 \\
9,52 \\
\end{array}$ & $\begin{array}{c}31 \\
(23,08) \\
\end{array}$ & $\begin{array}{c}5 \\
(9,26) \\
\end{array}$ \\
\hline Granada & $\begin{array}{c}14 \\
(7,51) \\
\end{array}$ & $\begin{array}{c}29 \\
(19,17) \\
\end{array}$ & $\begin{array}{c}19 \\
(32,76) \\
\end{array}$ \\
\hline La Coruña & $\begin{array}{c}6 \\
(2,93)\end{array}$ & & \\
\hline León & 0 & & 0 \\
\hline Murcia & $\begin{array}{c}10 \\
(5,32)\end{array}$ & $\begin{array}{c}20 \\
(11,38)\end{array}$ & $\begin{array}{c}6 \\
(12,50)\end{array}$ \\
\hline Salamanca & $\begin{array}{c}4,5 \\
(2,39)\end{array}$ & $\begin{array}{c}30 \\
(25)\end{array}$ & $\begin{array}{c}14 \\
(34,15)\end{array}$ \\
\hline Valencia & $\begin{array}{r}13,5 \\
(7,50) \\
\end{array}$ & & $\begin{array}{c}3 \\
(8,33) \\
\end{array}$ \\
\hline Vic & $\begin{array}{c}6 \\
(3,09)\end{array}$ & & \\
\hline
\end{tabular}


Tabla 8

Comparativa de créditos cuantitativos en las tres titulaciones

(Cont.)

\begin{tabular}{|l|c|c|c|}
\hline \multicolumn{1}{|c|}{ Universidad } & Diplomatura & Licenciatura & Doctorado \\
\hline Zaragoza & $\begin{array}{c}\text { (2,93) } \\
\text { AlcaládeHenares }\end{array}$ & & $\begin{array}{c}4 \\
(6,78)\end{array}$ \\
\hline $\begin{array}{l}\text { Autónoma de } \\
\text { Barcelona }\end{array}$ & & $(31,46)$ & 0 \\
\hline $\begin{array}{l}\text { Politécnica de } \\
\text { Valencia }\end{array}$ & 16,5 & \\
\hline UOC & & $(13,36)$ & \\
\hline
\end{tabular}

Lascifrassinparéntesisindicancréditosdemate riascuantitativasporuniversidades,yentreparénte sislos porcentajesdecréditoscuantitativosconrelaciónalacargalectiva

\section{CONCLUSIONES}

A pe sarde que la biblio me tría y las ma te rias de cor te cuan ti ta tivo en Es pa ña no tur vieronpresenciaenlosprimerosplanesdees tu diodelasuniversida desespañolas, la situación ha ido cambian do a me di da que se mo di fi ca ban los pla nes de es tu dio dela diplomatura y se incorporaba el segundociclo (licencia tu ra) y el tercero (doc to rado), lo quemarcóen ciertamedidauna orien ta ciónha cia el campodelas humanidades odelas cienciassocialesenlasdiferentesescuelasyfacultades.

Lain tro duc ción delos es tu dios mé tricos en ge ne ralen los pla nes de es tu diodelas escuelas y facultades de $\mathrm{ByD}$ ha sido un proceso paula ti no y está to da vía en fase de implantación.

La desigual distribución de materias cuantitativas y la categorización de éstas en las dis tintas titulaciones (diplomatura, licencia tu ra, doc to rado) muestran elbalance finaldel procesoderenovación em pren didoporlas diferentes es cuelas y facultades ensusplanesdeestudio.

Así pues, el ba lan ce que di lu ci da mos se ría que una de las ra zo nes de la fal ta de ho moge neiza ción enlas asigna tu ras de corte cuan tita tivo, se debe, en parte, a queno se tratade una mate ria tron caly a quela ten den cia ha sido ir in corporandomate riasobliga to rias y op ta ti vas en fun ción del per fil que se le haya que ri do dara la diplo ma tu ra; 
como el per fil hu ma nís tico que han teni do y man tienen en la di plo ma tu ra León, ZaragozaySalamanca.

Endiplo matu ralas universidades enlasquemás peso tienenlas materias cuantita tivasenrelaciónalacargalectivasonEx tremadura, GranadayValencia.

Enlalicencia tu ra seincremen ta el nú mero de asigna turas de cor te cuan ti ta tivoy prevalecenlasasignaturasobliga torias, a di ferenciadeladiplomatura, dondese da un claropredominiodelaoptatividad.

En el análisis comparativo de la carga lectiva asignada a las asignaturas de corte cuantitativo la licenciatura de las distintas universidades revela diferencias de unos planes respecto de otros. Las universidadesen las que más peso tienen las materias cuantitativas son Alcalá de Henares, Salamanca, Extremadura y Granada. Llama la atención que Salamanca no presentaba un peso significativo en la diplomatura por las razones expuestas anteriormente, y que en la licenciatura y su proyección en el doctoradoaparececonunagransensibilidadhacialosmétodoscuantitativos.

Sipartimos delabase de quelasmaterias de cor te cuan tita tivoes tán enunproceso de consolidación, esto implícitamente supone que hay un incremento del conocimientogenera doenellas, locualimplicaunincrementodelaactividadinvestigadora.

Elcientificismode una dis ciplinava apa reja doconla ac tividadinves tiga do ra.Una facetadeesaactividadsereflejaconlaobtención dela su ficienciainves tigadora (doc torado) y mediante la realización de la tesis doctoral que conduce al grado de doctor, comomáximotítulouniversitariootorga doporlasuniversidadesyquecapacitaaldocto rando paralainves tiga cióny para con tribuiralde sarrollodel conocimien to de nuestra disciplina. Es por ello que consideramos necesario potenciar el número de cursos decaráctercuantitativoeneltercerciclo.

Endoctorado (tercerciclo) las universidadesquehanoptadoporunmayornúmero de cursos de ca rác tercuan tita tivo son Sa la man cay Gra nada, y tres las universidadesqueno tienen pre sen cia delos mé to dos cuan titativos, Alca ládeHena res, León y la Complu ten se. Esta face ta delain ves tiga ción nos in dicauna vez más el tar dío de sa rrollodenuestraárea.

Esta desigual introducción de materias cuantitativas en los planes de estudio de ByD enEs pa ña cons ta ta el pro ce so deim ple men ta ción gra dual eina ca ba do que experimentaronlasuniversidadesespañolas desdelareformadelosplanesdeestudio.

Portan to sehacein dis pen sableque territoriosinterme dios en trelas cien cias ylasletras seancul tiva dos por pro fe sio na les que reú nan ambas forma ciones para no caeren gra ves erro res en cuan to a fi na li dad o me to do lo gía. Por otra par te es cada vez más nece sario porpartedel biblio te cario o do cumen talis ta tenercono cimien tos so bre parámetrosyva riables rela cio na dos conla producción científica, sucircula cióny suconsumo,yapostarporlapromocióndeestudios cuantitativosenlaenseñanzadeByD. 


\section{BIBLIOGRAFÍA}

[1]. Aba dal Fal gue ras, E. (1994).LaDocumentaciónenEspaña. Ma drid:CINDOC.

[2]. Amat Noguera, Nuria (1991). "El Documentalista: un científico de científicos". En RevistaEspañoladeDocumentación Científica, vol. 14, n. 2, p. 179-186.

[3].Apa ricioFernández,M.d.C.(1990). La formación de los biblioteca riosen Es paña. Seminariobispano-británicosobrecooperacióneinformatizaciónbibliotecaria.

[4]. Camps, E.; Crespán, J.L. (1997). “Una profesiónobligada a cambiar. Los bibliotecarios-documentalistas en Cataluña: situación actual y perspectivas de futuro" En: Revista General de Información y Documentación., vol. 7, no 1, p. 299-311.

[5]. Cro nin, Blai se (1992). In for ma tion scien ce in the in ter na tio nal are na: an educationalpers pective. As lib Proceedings, vol.44,n.4,p.195-202.

[6]. Curras, E. (1993). "The need for theoretical studies in Information Science”. Journal of Information Science, vol. 19, n.1.

[7]. DelgadoLó pez-Cozar,E.(1992a). “La reforma delas en señan zasuniversita rias enEs paña: unas nuevas direc trices para el títulode diplomado”. En: Biblioteconomía y Documentación. Boletín de la Asociación Andaluza de Bibliotecarios, n.33, p. 5-22.

[7]. Delga do Ló pez-Co zar,E. (1992b). "Prin cipios, es truc tu ra y con tenidos de un programa de pro fe sionales dela In for ma ción y Documen tación: propuesta del plan de estudios para la EUBD de Granada". En: Boletín de la Asociación Andaluza de Bibliotecarios, n. 30, p. 5-35.

[8].DelgadoLópez-Cozar,E.(2000). “Diagnós ticodelainves tigación en BiblioteconomíayDocumentación enEspaña(1976-1996)"En: Revista de Investigación Iberoamericanaen Cienciadela Informacióny Documentación, vol. 1, n. 1, p. 79-93.

[10]. Delgado López-Cozar (2002). La investigaciónen biblioteconomía y documentación. Gijón: Trea.

[9]. Ferreiro Alaez, L. (1987). "Didáctica bibliométrica: el concepto instrumental de logaritmo". En: Revista española de Documentación cientifica, vol. 10, n. 1, p. 11-27.

[10]. Ferreiro Aláez, L. (1990). "Car ta al di rec tor". En:Revista Española de Documentación Cientifica, vol. 13, n. 1, p. 651-52.

[11]. Frías, J.A.; Ro me ro Gó mez, P. (1998). “¿Qui enes son y qué ci tan los investigadores que publican en las revistas españolas de Biblioteconomía y Documentación?” En: Anales de Documentación, n. 1, p. 29-53.

[12]. Gorbea-Por tal, Salva dor(1994). "Principios teó ricos y me to dológi cos delos es tu dios mé tri cos delain for ma ción”. En: InvestigaciónBibliotecológica: Archivonomia, Bibliotecología e Información, vol. 8, n. 17, p. 23-32. 
[13]. Gorman, G. E. (1999). "The future for library science education". En Libri, vol. 49, n. 1, p. 1-10.

[16]. Ingwersen, Peter (1994). "The human approach to information science and management: the framework and prospect underlying the new Da nish msc pro gram me". En: JournalofInformation Science, vol. 20, n. 3, p. $91-98$

[17]. Johnson, L. M. (1998). "Challenges in developing profesionals for the 'info rm ation so ciety': and some res pon ses by the bri tish schools of libra rianship and forma tion stu dies". En: Library Review, vol. 47, n. 3, p. 52-59.

[18].JiménezContreras,E.(1990). "Notas en tornoala fu turalicencia tura en Ciencias de la Documentación”. En: Revista Española de Documentación Científica, vol. 13, n. 4, p. 631-35.

[19]. Jiménez Contreras, E. (1997). Proyecto docente. Granada.

[20]. Jiménez-Contreras, Evaristo; Pulgarín-Guerrero, Antonio (1998). "Bibliometrics-Informetrics and other quantitative subjets in library and information science curricula in Spain". En: Education for Information, vol. 16, p. 341-355.

[21].Ló pezLópez,P.(1996). "Lainves tiga ción bibliométricaenEs paña”. En: Revista Española de Documentación Científica, vol.19, n. 1, p. 85-89.

[22]. López Yepes, J. (1995). “La licenciatura en documentación, marco formativo de un nuevoprofesional". En: RevistaGeneral de Información y Documentación, vol. 5, n. 1, p. 33-69.

[23].López Yepes,J.(1996).Sobre forma ción pro fe sionaly problemática

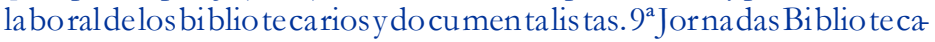
rias en Andalucia. Granada: Asociación Andaluza de Bibliotecarios: Diputación Provincial, p. 277-298.

[24]. Ló pez Yepes, José(2001). "Inves tiga do res y direc to res de te sis docto rales, crea do res de fo cos deinves tiga ción y de es cuelas cien tíficas en Documentación. Elcaso delDepartamen to de Biblio te co no míay Documentación de la Universidad Complutense de Madrid". En: V Congreso Isko-España, 25-27 de abril de 2001, Alcalá de Henares. Alcalá de Henares, Madrid: Universidad, Facultad de Documentación.

[25]. Meyriat, Jean (1993). "La formation initiale en France des profession nels del'in for ma tion et de la do cui men ta tion". En: Documentaliste, vol. 30, n. 2, p.91-98

[26]. Moreiro González, J.A.; Moscoso Castro, P.; Ortiz Repiso, V. (1995). "Elmerca do de tra ba jo de los diplo ma dos es pañoles en biblioteconomía y documentación”. En: Revista Española de Documentación Cientifica, vol. 18, n. 4, p. 444-463. 
[27]. Moreiro, J. A.; Caridad, M. (1998). “Acer ca de los mé to dos de es tudiodela rela ción en trelas con dicioneslaborales y forma tivas en Biblioteconomía y Documentación. El caso de la Universidad Carlos III de Madrid". En: Anales de Documentación, v. 1, p. 137-153.

[28]. Moya Anegón, Félix (1996). "La ca li dad de los servi cios en la so ciedaddelain for ma ción y la forma ción de los fu tu ros pro fe sio nales". En: Revista General de Información y Documentación, vol. 6, n. 1, p. 241-256.

[29]. Moya Anegón, Felix de.; Jimenez Contreras, E.; Moneda, M. de la (1998). "Research fronts in Library and Information Science in Spain (1985-1994)" En: Scientometrics, vol. 42, n. 2, p. 229-246.

[30]. Pérez Álvarez-Ossorio, J. R. (1997). “Cobertura temática y procedenciainstitucional de los artículos publica dos enla Revista Española de Documentación Científica en sus vein te años de exis ten cia”. En: Revista Es pañola de Documentación Científica, vol. 20, n. 4, p. 290-298.

[31]. Prevot, Mar tine (1990). "Les for ma tions eu ro pée nes en scien ces de l'information En: Documentaliste, vol. 27, n. 4-5, p. 186-192.

[32]. Reyes-Barragán,María J. (1996). “Justificación de la bibliometríaen la enseñanza universitaria de Biblioteconomía y Documentación: una experienciadocente". En: Actas de las IXJornadasBibliotecarias de An dalut cía: Granada, 1996. Granada: Aso cia ción AndaluzadeBibliotecarios,p. 377-385. ISBN 84-9209-14-0

[33]. Ro man, A; Sorli, A. (1994). “La do cu men ta ción en los 90.¿Pod emos pre de cir el fu tu ro ras trean do el pa sa do re cien te dela in ves tiga ción do cumental?" En: III Jornadas españolas de Documentación Automatizada, p. 1171-1185. Palma de Mallorca: Universitat de Iles Balears.

[34]. Sanz Ca sa do, Elías et al. (2001). "Las dis ci pli nas cien tí fi cas como encru cija da de sa be res: el caso de los es tu dios de biblio te co no mía y do cur mentación de las Universidades españolas". En: Revista General de Información y Documentación, vol. 11, n. 1, p. 175-189.

[35]. Sebastia Salat, M. (1992). "De la invisibilidad a la visibilidad. Reflexio nes so bre la for ma ción de es pe cia lis tas y usua rios de la TI”. En:Revista Española de Documentación Cientifica, vol. 15, n. 1, p. 31-43.

[36].ZapicoAlon so, Felipe; Reyes-Ba rragán,María J.; GuerreroBote, Vi cente; López Pujalte, Cristina (2002). "La investigación en Documentación: análisis bibliométrico de las tesis en Documentación”. En: $\mathrm{M}^{\mathrm{a}}$ Antonia Morán Suárez, $\mathrm{M}^{\mathrm{a}}$ del Carmen Rodríguez López (coord.). La documentación para la investigación: homenaje a José Antonio Martín Fuertes. León: Universidad, 2 vol., p. 691-713.

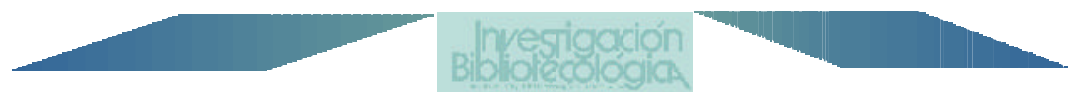

